\title{
On hyper-exponential output-feedback stabilization of a double integrator by using artificial delay
}

\author{
Efimov D., Fridman E., Perruquetti W., Richard J.-P.
}

\begin{abstract}
The problem of output-feedback stabilization of a double integrator is revisited with the objective of achieving the rates of convergence faster than exponential. It is assumed that only position is available for measurements, and the designed feedback is based on the output and its delayed values without an estimation of velocity. It is shown that by selecting the closedloop system to be homogeneous with negative or positive degree it is possible to accelerate the rate of convergence in the system at the price of a small steady-state error. Efficiency of the proposed control is demonstrated in simulations.
\end{abstract}

\section{INTRODUCTION}

The design of regulators for dynamical systems is a fundamental and complex problem studied in the control theory. An important feature of different existing methods for control synthesis is the achievable quality of transients and robustness against exogenous perturbations and noises. Very frequently the design methods are oriented on various canonical models, and the linear ones are the most popular. Then the double integrator is a conventional benchmark system, since the tools designed for it can be easily extended to other more generic models. If non-asymptotic rates of convergence (i.e. finite-time or fixed time [1]) are needed in the closed-loop system, then usually homogeneous systems come to the attention as canonical dynamics, which include linear models as a subclass.

The theory of homogeneous dynamical systems is welldeveloped for continuous time-invariant differential equations [2], [3], [4], [5] or time-delay systems [6], [7] (applications of the conventional homogeneity theory to analysis of timedelay systems considering delay as a kind of perturbation have been considered in [8], [9], [10], [11]). The main feature of a homogeneous system (described by ordinary differential equation) is that its local behavior of trajectories is the same as global (local attractiveness implies global asymptotic stability, for example [1]), while for time-delay homogeneous

D. Efimov, W. Perruquetti and J.-P. Richard are at Inria, Non-A team, Parc Scientifique de la Haute Borne, 40 av. Halley, 59650 Villeneuve d'Ascq, France and CRIStAL (UMR-CNRS 9189), Ecole Centrale de Lille, BP 48, Cité Scientifique, 59651 Villeneuve-d'Ascq, France.

E. Fridman is with School of Electrical Engineering, Tel-Aviv University, Tel-Aviv 69978, Israel.

D. Efimov is with Department of Control Systems and Informatics, Saint Petersburg State University of Information Technologies Mechanics and Optics (ITMO), 49 Kronverkskiy av., 197101 Saint Petersburg, Russia.

This work was partially supported by the Government of Russian Federation (Grant 074-U01), the Ministry of Education and Science of Russian Federation (Project 14.Z50.31.0031) and by Israel Science Foundation (grant No $1128 / 14)$. systems the independent on delay (IOD) stability follows [7], with certain robustness to exogenous inputs in both cases. The rate of convergence for homogeneous ordinary differential equations is related with degree of homogeneity [1], but for time-delay systems the links are not so straightforward [12]. In addition, the homogeneous stable/unstable systems admit homogeneous Lyapunov functions [5], [13], [14].

Analysis of delay influence on the system stability is vital in many cases [15], [16]. Despite of variety of applications, most of them deal with the linear time-delay models, which is originated by complexity of stability analysis for time-delay systems [16]. However, in some cases introduction of a delay may lead to an improvement of the system performance [17], [18].

The goal of this work is to develop the results obtained in [17], [18] for linear systems to a nonlinear homogeneous case restricting for brevity the attention to the case of the double integrator model. A design method is proposed, which uses position and its delayed values for practical output stabilization with hyper-exponential convergence rates.

The outline of this work is as follows. The preliminary definitions and homogeneity concept for time-delay systems are given in Section II. The problem statement and the control design and stability analysis are presented in sections III and IV, respectively. An example is considered in Section V.

\section{PRELIMINARIES}

Consider an autonomous functional differential equation of retarded type with inputs [19]:

$$
\dot{x}(t)=f\left(x_{t}, d(t)\right), t \geq 0
$$

where $x(t) \in \mathbb{R}^{n}$ and $x_{t} \in C_{[-\tau, 0]}$ is the state function (we denote by $C_{[a, b]}, 0 \leq a<b \leq+\infty$ the Banach space of continuous functions $\phi:[a, b] \rightarrow \mathbb{R}^{n}$ with the uniform norm $\|\phi\|=\sup _{a \leq \varsigma \leq b}|\phi(\varsigma)|$, where $|\cdot|$ is the standard Euclidean norm $), x_{t}(s) \stackrel{=}{=} x(t+s),-\tau \leq s \leq 0 ; d(t) \in \mathbb{R}^{m}$ is the external input, it is a Lebesgue measurable essentially bounded function of time $d: \mathbb{R}_{+} \rightarrow \mathbb{R}^{m}$ with the norm $\|d\|_{\left[t_{0}, t_{1}\right)}=\operatorname{ess} \sup _{t \in\left[t_{0}, t_{1}\right)}\|d(t)\|$, then $\|d\|_{\infty}=\|d\|_{[0,+\infty)}$ and the set of $d(t)$ with the property $\|d\|_{\infty}<+\infty$ we further denote as $\mathcal{L}_{\infty}^{m} ; f: C_{[-\tau, 0]} \times \mathbb{R}^{m} \rightarrow \mathbb{R}^{n}$ is a continuous function ensuring forward uniqueness and existence of the system solutions, $f(0,0)=0$. We assume that for the initial functional condition $x_{0} \in C_{[-\tau, 0]}$ and $d \in \mathcal{L}_{\infty}^{m}$ the system 
(1) admits a unique solution $x\left(t, x_{0}, d\right)$, which is defined on some time interval $[-\tau, T)$ for $T>0$.

The upper right-hand Dini derivative of a locally Lipschitz continuous functional $V: C_{[-\tau, 0]} \rightarrow \mathbb{R}_{+}$along the system (1) solutions is defined as follows for any $\phi \in C_{[-\tau, 0]}$ and $d \in \mathbb{R}^{m}$ :

$$
D^{+} V(\phi, d)=\lim _{h \rightarrow 0^{+}} \sup \frac{1}{h}\left[V\left(\phi_{h}\right)-V(\phi)\right],
$$

where $\phi_{h} \in C_{[-\tau, 0]}$ for $0<h<\tau$ is given by

$$
\phi_{h}= \begin{cases}\phi(\theta+h), & \theta \in[-\tau,-h) \\ \phi(0)+f(\phi, d)(\theta+h), & \theta \in[-h, 0] .\end{cases}
$$

A continuous function $\sigma: \mathbb{R}_{+} \rightarrow \mathbb{R}_{+}$belongs to class $\mathcal{K}$ if it is strictly increasing and $\sigma(0)=0$; it belongs to class $\mathcal{K}_{\infty}$ if it is also radially unbounded. A continuous function $\beta: \mathbb{R}_{+} \times \mathbb{R}_{+} \rightarrow \mathbb{R}_{+}$belongs to class $\mathcal{K} \mathcal{L}$ if $\beta(\cdot, r) \in \mathcal{K}$ and $\beta(r, \cdot)$ is a strictly decreasing to zero for any fixed $r \in \mathbb{R}_{+}$.

The symbol $\overline{1, m}$ is used to denote a sequence of integers $1, \ldots, m$. For a symmetric matrix $P \in \mathbb{R}^{n \times n}$, the minimum and maximum eigenvalues are denoted as $\lambda_{\min }(P)$ and $\lambda_{\max }(P)$, respectively.

\section{A. ISS of time delay systems}

The input-to-state stability (ISS) property is an extension of conventional stability paradigm to the systems with external inputs [20], [21].

Definition 1. [20] The system (1) is called ISS, if for all $x_{0} \in C_{[-\tau, 0]}$ and $d \in \mathcal{L}_{\infty}^{m}$ the solutions are defined for all $t \geq 0$ and there exist $\beta \in \mathcal{K} \mathcal{L}$ and $\gamma \in \mathcal{K}$ such that

$$
\left|x\left(t, x_{0}, d\right)\right| \leq \beta\left(\left\|x_{0}\right\|, t\right)+\gamma\left(\|d\|_{\infty}\right) \quad \forall t \geq 0 .
$$

Definition 2. [20] A locally Lipschitz continuous functional $V: C_{[-\tau, 0]} \rightarrow \mathbb{R}_{+}$is called ISS Lyapunov-Krasovskii functional for the system (1) if there exist $\alpha_{1}, \alpha_{2} \in \mathcal{K}_{\infty}$ and $\alpha, \chi \in \mathcal{K}$ such that for all $\phi \in C_{[-\tau, 0]}$ and $d \in \mathbb{R}^{m}$ :

$$
\begin{gathered}
\alpha_{1}(|\phi(0)|) \leq V(\phi) \leq \alpha_{2}(\|\phi\|), \\
V(\phi) \geq \chi(|d|) \Longrightarrow D^{+} V(\phi, d) \leq-\alpha(V(\phi)) .
\end{gathered}
$$

Theorem 1. [20] If there exists an ISS Lyapunov-Krasovskii functional for the system (1), then it is ISS with $\gamma=\alpha_{1}^{-1} \circ \chi$.

\section{B. Homogeneity}

For any $r_{i}>0, i=\overline{1, n}$ and $\lambda>0$, define the dilation matrix $\Lambda_{r}(\lambda)=\operatorname{diag}\left\{\lambda^{r_{i}}\right\}_{i=1}^{n}$ and the vector of weights $r=$ $\left[r_{1}, \ldots, r_{n}\right]^{T}$.

Definition 3. [22] The function $g: C_{[-\tau, 0]} \rightarrow \mathbb{R}$ is called $r$-homogeneous $\left(r_{i}>0, i=\overline{1, n}\right)$, if for any $\phi \in C_{[-\tau, 0]}$ the relation

$$
g\left(\Lambda_{r}(\lambda) \phi\right)=\lambda^{\nu} g(\phi)
$$

holds for some $\nu \in \mathbb{R}$ and all $\lambda>0$.
The vector field $f: C_{[-\tau, 0]} \rightarrow \mathbb{R}^{n}$ is called $r$ homogeneous $\left(r_{i}>0, i=\overline{1, n}\right)$, if for any $\phi \in C_{[-\tau, 0]}$ the relation

$$
f\left(\Lambda_{r}(\lambda) \phi\right)=\lambda^{\nu} \Lambda_{r}(\lambda) f(\phi)
$$

holds for some $\nu \geq-\min _{1 \leq i \leq n} r_{i}$ and all $\lambda>0$.

In both cases, the constant $\nu$ is called the degree of homogeneity.

The introduced notion of homogeneity in $C_{[-\tau, 0]}$ is reduced to the standard one in $\mathbb{R}^{n}$ [5] under a vector argument substitution.

For any $x \in \mathbb{R}^{n}$ the homogeneous norm can be defined as follows

$$
|x|_{r}=\left(\sum_{i=1}^{n}\left|x_{i}\right|^{\varrho / r_{i}}\right)^{1 / \varrho}, \varrho \geq \max _{1 \leq i \leq n} r_{i} .
$$

For all $x \in \mathbb{R}^{n}$, its Euclidean norm $|x|$ is related with the homogeneous one:

$$
\underline{\sigma}_{r}\left(|x|_{r}\right) \leq|x| \leq \bar{\sigma}_{r}\left(|x|_{r}\right)
$$

for some $\underline{\sigma}_{r}, \bar{\sigma}_{r} \in \mathcal{K}_{\infty}$. The homogeneous norm is a $r$ homogeneous function of degree one: $\left|\Lambda_{r}(\lambda) x\right|_{r}=\lambda|x|_{r}$ for all $x \in \mathbb{R}^{n}$. Similarly, for any $\phi \in C_{[a, b]},-\infty \leq a<b \leq$ $+\infty$ the homogeneous norm can be defined as follows

$$
\|\phi\|_{r}=\left(\sum_{i=1}^{n}\left\|\phi_{i}\right\|^{\varrho / r_{i}}\right)^{1 / \varrho}, \varrho \geq \max _{1 \leq i \leq n} r_{i},
$$

and there exist two functions $\underline{\rho}_{r}, \bar{\rho}_{r} \in \mathcal{K}_{\infty}$ such that for all $\phi \in C_{[a, b]}$

$$
\underline{\rho}_{r}\left(\|\phi\|_{r}\right) \leq\|\phi\| \leq \bar{\rho}_{r}\left(\|\phi\|_{r}\right)
$$

The homogeneous norm in the Banach space has the same homogeneity property that is $\left\|\Lambda_{r}(\lambda) \phi\right\|_{r}=\lambda\|\phi\|_{r}$ for all $\phi \in C_{[a, b]}$. In $C_{[-\tau, 0]}$, for a radius $\rho>0$, denote the corresponding sphere $\mathcal{S}_{\rho}^{\tau}=\left\{\phi \in C_{[-\tau, 0]}:\|\phi\|_{r}=\rho\right\}$ and the closed ball $B_{\rho}^{\tau}=\left\{\phi \in C_{[-\tau, 0]}:\|\phi\|_{r} \leq \rho\right\}$.

An advantage of homogeneous systems described by nonlinear ordinary differential equations is that any of its solution can be obtained from another solution under the dilation re-scaling and a suitable time parameterization. A similar property holds for functional homogeneous systems.

Proposition 1. [7] Let $x\left(t, x_{0}\right)$ be a solution of the $r$ homogeneous system

$$
d x(t) / d t=f\left(x_{t}\right), t \geq 0, x_{t} \in C_{[-\tau, 0]}
$$

with the degree $\nu$ for an initial condition $x_{0} \in C_{[-\tau, 0]}, \tau \in$ $(0,+\infty)$. For any $\lambda>0$ the functional differential equation

$$
d y(t) / d t=f\left(y_{t}\right), t \geq 0, y_{t} \in C_{\left[-\lambda^{-\nu} \tau, 0\right]}
$$

has a solution $y\left(t, y_{0}\right)=\Lambda_{r}(\lambda) x\left(\lambda^{\nu} t, x_{0}\right)$ with the initial condition $y_{0} \in C_{\left[-\lambda^{-\nu} \tau, 0\right]}, y_{0}(s)=\Lambda_{r}(\lambda) x_{0}\left(\lambda^{\nu} s\right)$ for $s \in$ $\left[-\lambda^{-\nu} \tau, 0\right]$. 
In [6], using that result it has been shown that for (3) with $\nu=0$ the local asymptotic stability implies global one (for the ordinary differential equations even more stronger conclusion can be obtained: local attractiveness implies global asymptotic stability [1]). For time-delay systems with $\nu \neq 0$ that result has the following correspondences:

Lemma 1. [7] Let the system (3) be r-homogeneous with degree $\nu \neq 0$ and globally asymptotically stable for some delay $0<\tau_{0}<+\infty$, then it is globally asymptotically stable for any delay $0<\tau<+\infty$ (i.e. IOD).

Corollary 1. [7] Let the system (3) be r-homogeneous with degree $\nu$ and asymptotically stable with the region of attraction $B_{\rho}^{\tau}$ for some $0<\rho<+\infty$ for any value of delay $0 \leq \tau<+\infty$, then it is globally asymptotically stable IOD.

Corollary 2. [7] Let the system (3) be r-homogeneous with degree $\nu<0$ and asymptotically stable with the region of attraction $B_{\rho}^{\tau}$ for some $0<\rho<+\infty$ for any value of delay $0 \leq \tau \leq \tau_{0}$ with $0<\tau_{0}<+\infty$, then it is globally asymptotically stable IOD.

Corollary 3. [7] Let the system (3) be r-homogeneous with degree $\nu>0$ and the set $B_{\rho}^{\tau}$ for some $0<\rho<+\infty$ be uniformly globally asymptotically stable for any value of delay $0 \leq \tau \leq \tau_{0}, 0<\tau_{0}<+\infty^{1}$, then (3) is globally asymptotically stable (at the origin) IOD.

\section{Problem StATEMEnT}

Consider the double integrator system:

$$
\begin{aligned}
\dot{x}_{1}(t) & =x_{2}(t), \\
\dot{x}_{2}(t) & =u(t), \\
y(t) & =x_{1}(t),
\end{aligned}
$$

where $x_{1}(t) \in \mathbb{R}$ and $x_{2}(t) \in \mathbb{R}$ are the position and velocity, respectively, $u(t) \in \mathbb{R}$ is the control input and $y(t) \in \mathbb{R}$ is the output available for measurements. The goal is to design a static output-feedback control practically stabilizing the system with a hyper-exponential convergence rate, i.e. with a convergence faster than any exponential.

\section{MAIN RESUlTS}

The solution considered in this paper is the delayed nonlinear controller

$$
u(t)=-\left(k_{1}+k_{2}\right)\lceil y(t)\rfloor^{\alpha}+k_{2}\lceil y(t-h)\rfloor^{\alpha},
$$

where $\lceil y\rfloor^{\alpha}=|y|^{\alpha} \operatorname{sign}(y), k_{1}>0$ and $k_{2}>0$ are tuning gains, $\alpha>0, \alpha \neq 1$ is a tuning power and $h>0$ is the delay (if $\alpha=1$ then the control (6) is linear and it has been studied in [17], [18]). The restrictions on selection of

\footnotetext{
${ }^{1}$ In this case for any $0 \leq \tau \leq \tau_{0}$, any $\varepsilon>0$ and $\kappa \geq 0$ there is $0 \leq T_{\kappa, \tau}^{\varepsilon}<+\infty$ such that $\left|x\left(t, x_{0}\right)\right|_{r} \leq \rho+\varepsilon$ for all $t \geq \bar{T}_{\kappa, \tau}^{\varepsilon}$ for any $x_{0} \in B_{\kappa}^{\tau}$, and $\left|x\left(t, x_{0}\right)\right|_{r} \leq \sigma_{\tau}\left(\left\|x_{0}\right\|_{r}\right)$ for all $t \geq 0$ for some function $\sigma_{\tau} \in \mathcal{K}_{\infty}$ for all $x_{0} \in C_{[-\tau, 0]}$.
}

these parameters and the conditions to check are given in the following theorem.

Theorem 2. For any $k_{1}>0, k_{2}>0, h_{0}>0$, if the system of linear matrix inequalities

$$
\begin{gathered}
Q \leq 0, P>0, q>0, \\
Q=\left[\begin{array}{ccc}
Q_{11} & k_{2} Z b & Z b \\
k_{2} b^{\top} Z^{\top} & \left(k_{2}^{2} h^{2}-4 \frac{e^{-\varpi h}}{h^{2}}\right) q & q h^{2} k_{2} \\
b^{\top} Z^{\top} & q h^{2} k_{2} & q h^{2}-\gamma
\end{array}\right], \\
Q_{11}=A^{\top} P+P A+q h^{2} A^{\top} b b^{\top} A+\varpi P, \\
Z=P+q h^{2} A^{\top}, \\
A=\left[\begin{array}{cc}
0 & 1 \\
-k_{1} & -k_{2} h
\end{array}\right], b=\left[\begin{array}{l}
0 \\
1
\end{array}\right]
\end{gathered}
$$

is feasible for some $\varpi>0, \gamma>0$ and any $0<h \leq h_{0}$, then for any $0<\eta<+\infty$ there exists $\epsilon \in(0,1)$ sufficiently small such that the system (5), (6) is

a) globally asymptotically stable with respect to the set $B_{\eta}^{2 h}$ for any $\alpha \in(1-\epsilon, 1)$;

b) locally asymptotically stable at the origin from $B_{\eta}^{2 h}$ for any $\alpha \in(1,1+\epsilon)$.

All proofs are omitted due to space limitations. Note that for any $\alpha \geq 0$ the closed-loop system (5), (6) is $r$ homogeneous for $r_{1}=1$ and $r_{2}=\frac{\alpha+1}{2}$ with the degree $\nu=\frac{\alpha-1}{2}$, then the result of Proposition 1 can be used for substantiation.

The requirement that the matrix inequalities (7) have to be verified for any $0<h \leq h_{0}$ may be restrictive for given gains $k_{1}$ and $k_{2}$, then another local result can be obtained by relaxing this constraint.

Corollary 4. For any $k_{1}>0, k_{2}>0$ and $0<h_{1}<h_{0}$, let the system of linear matrix inequalities (7) be verified for some $\varpi>0$ and all $h_{1} \leq h \leq h_{0}$. Then for any $0<\rho_{1}<$ $+\infty$ there exist $\epsilon \in(0,1)$ sufficiently small and $\rho_{2}>\rho_{1}$ such that the system (5), (6) is asymptotically stable with respect to the set $B_{\rho_{1}}^{2 h}$ with the region of attraction $B_{\rho_{2}}^{2 h}$ for any $\alpha \in(1-\epsilon, 1+\epsilon)$.

Remark 1. The result of Theorem 2 complements corollaries 2 and 3.

Note that in all cases, for $\nu \neq 0$, the global stability at the origin cannot be obtained in (5), (6) (due to homogeneity of the system, following the result of Lemma 1 the globality implies IOD result), while in the linear case with $\nu=0$ such a result is possible to derive for any $0<h \leq h_{0}$. Then it is necessary to justify a need in the control with $\nu \neq 0$ comparing to the linear feedback with the same gains. An answer to this question is presented in the following result, and to this end denote for the system (5), (6):

$$
\mathcal{T}\left(\alpha, \rho_{1}, \rho_{2}, h\right)=\underset{t \geq T-h}{\arg \sup } \sup _{x_{0} \in \mathcal{S}_{\rho_{2}}^{2 h}}\left|x\left(t, x_{0}\right)\right|_{r} \leq \rho_{1}
$$


as the time of convergence of all trajectories initiated on the sphere $\mathcal{S}_{\rho_{2}}^{2 h}$ to the set $B_{\rho_{1}}^{2 h}$ provided that the delay $h$ and the power $\alpha$ applied in the feedback.

Proposition 2. For given $k_{1}>0, k_{2}>0, h_{0}>0$, let the system of linear matrix inequalities (7) be verified for some $\varpi>0$ and any $0<h \leq h_{0}$. Then there exist $\epsilon \in(0,1)$ sufficiently small and $0<\rho_{1}<\rho_{2}<+\infty$ such that in the system (5), (6)

$$
\mathcal{T}\left(\alpha, \rho_{1}, \rho_{2}, h^{\prime}\right)<\mathcal{T}\left(1, \rho_{1}, \rho_{2}, h^{\prime}\right)
$$

for some $0<h^{\prime} \leq h_{0}$ and any $\alpha \in(1-\epsilon, 1)$ or $\alpha \in(1,1+\epsilon)$, provided that

$$
\sup _{0<h \leq h_{0}} \mathcal{T}(\alpha, 0.5,1, h) \leq T_{\alpha}
$$

for some $T_{\alpha} \in \mathbb{R}_{+}$and all $\alpha \in(1-\epsilon, 1+\epsilon)$.

In other words, the result above claims that for any fixed feedback gains $k_{1}$ and $k_{2}$, if the conditions of Theorem 2 are satisfied, then the nonlinear closed-loop system (5), (6) with $\nu \neq 0(\alpha \neq 1)$ is always converging faster than its linear analog with $\nu=0(\alpha=1)$ between properly selected levels $\rho_{1}$ and $\rho_{2}$ (which values depend on smaller or high than 1 is $\alpha$ ) for a delay $h^{\prime}$.

The result of Proposition 2 provides a motivation for using nonlinear control in this setting: playing with degree of homogeneity of the closed-loop system it is possible to accelerate the obtained linear feedback by fixing the gains and delay values, but introducing an additional power tuning parameter. Note that another, conventional solution, which consists in gains $k_{1}$ and $k_{2}$ increasing for acceleration, may be infeasible for the given delay value $h_{0}$. Let us consider some results of application of the proposed control and an illustration of the obtained acceleration.

\section{EXAMPLE}

Take

$$
h_{0}=0.3, k_{1}=0.25, k_{2}=0.1, \varpi=10^{-5},
$$

then for

$$
\begin{gathered}
P=10^{-8} \times\left[\begin{array}{cc}
0.1 & 0.00 \\
0.00 & 0.4
\end{array}\right], \gamma=3.73 \times 10^{-2}, \\
q=1.5 \times 10^{-11}
\end{gathered}
$$

the matrix inequalities (7) are satisfied for $h_{1}<h \leq h_{0}$ with $h_{1}=5 \times 10^{-4}$, and the results of verification are presented in Fig. 1. Thus, all conditions of Corollary 4 are verified. The errors of regulation obtained in simulation of the system (5), (6) with delay $h_{0}$ for different initial conditions with $\alpha=0.8$ and $\alpha=1.2$, in comparison with the linear controller with $\alpha=1$, are shown in figures 2 and 3 , respectively (the solid lines represent the trajectories of the system with $\alpha \neq 1$ and the dashed ones correspond to $\alpha=1$, since the plots are given in a logarithmic scale, then the latter trajectories are close to straight lines). As we can conclude, in the nonlinear

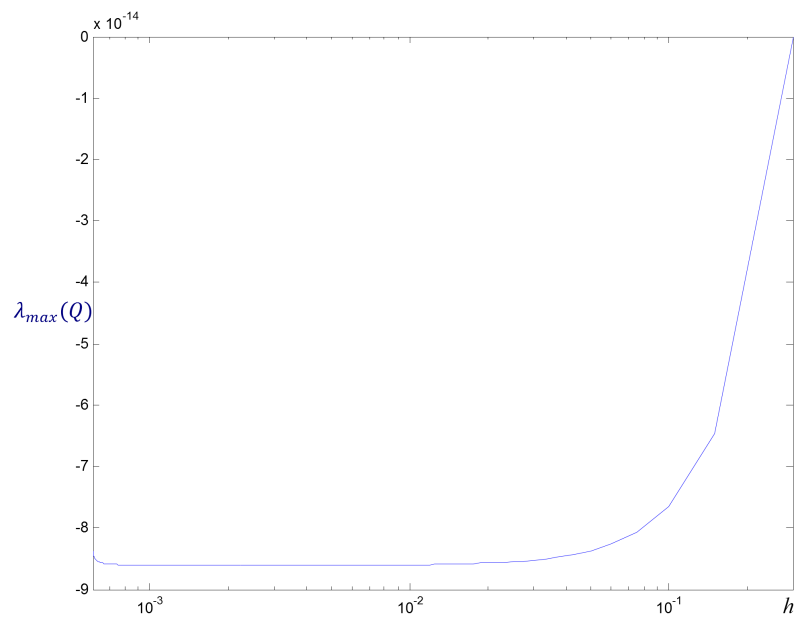

Figure 1. The results of verification of (7) for different $h$

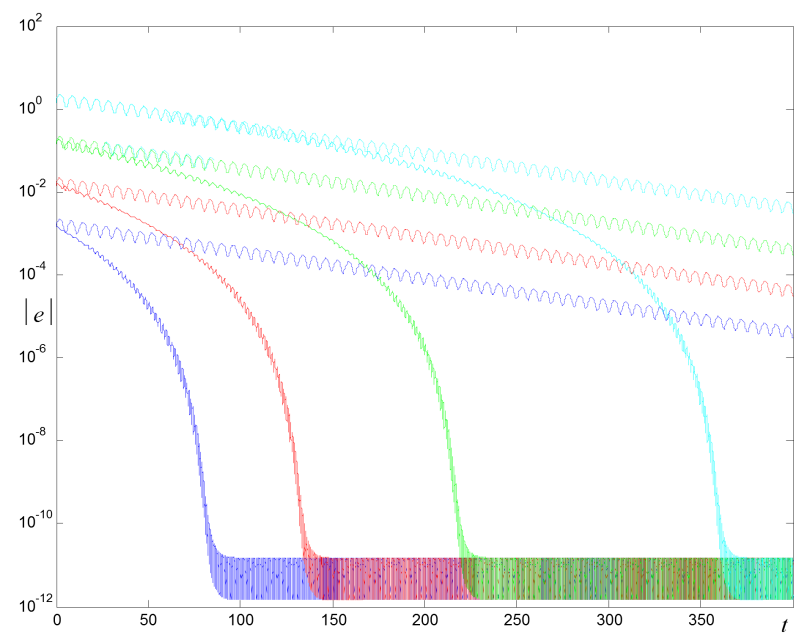

Figure 2. Trajectories of stabilized double integrator with $\alpha=0.8$

case the convergence is much faster than in the linear one close to the origin for $\alpha \in(0,1)$ and far outside for $\alpha>1$, which confirms the statement of Proposition 2. Note that the value of $\eta$ (the radius of the set to which the trajectories converge for $\alpha<1$ or from which they converge to the origin for $\alpha>1$ ) is not restrictive.

\section{CONCLUSIONS}

The paper addresses the problem of output stabilization of the double integrator using a nonlinear delayed feedback by obtaining hyper-exponential (faster than any exponential) rates of convergence. The control does not need an estimation of velocity, and the applicability of the approach can be checked by resolving linear matrix inequalities. The efficiency of the proposed approach is demonstrated in simulations and a comparison with a linear controller is carried out. 


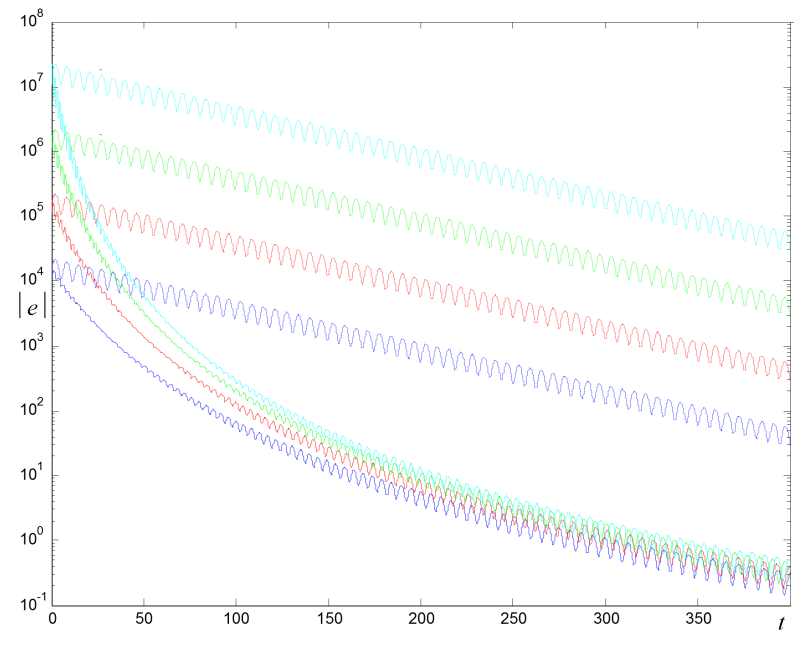

Figure 3. Trajectories of stabilized double integrator with $\alpha=1.2$

\section{REFERENCES}

[1] E. Bernuau, D. Efimov, W. Perruquetti, and A. Polyakov, "On homogeneity and its application in sliding mode," International Journal of Franklin Institute, vol. 351, no. 4, pp. 1866-1901, 2014.

[2] A. Bacciotti and L. Rosier, Liapunov Functions and Stability in Control Theory, vol. 267 of Lecture Notes in Control and Inform. Sci. Berlin: Springer, 2001.

[3] S. Bhat and D. Bernstein, "Geometric homogeneity with applications to finite-time stability," Mathematics of Control, Signals and Systems, vol. 17, pp. 101-127, 2005.

[4] M. Kawski, Homogeneous feedback stabilization, vol. 7 of Progress in systems and control theory: New trends in systems theory. Birkhäuser, 1991.

[5] V. Zubov, "On systems of ordinary differential equations with generalized homogenous right-hand sides," Izvestia vuzov. Mathematica., vol. 1, pp. 80-88, 1958. in Russian.

[6] D. Efimov, W. Perruquetti, and J.-P. Richard, "Development of homogeneity concept for time-delay systems," SIAM J. Control Optim., vol. 52, no. 3, pp. 1403-1808, 2014.

[7] D. Efimov, A. Polyakov, W. Perruquetti, and J.-P. Richard, "Weighted homogeneity for time-delay systems: Finite-time and independent of delay stability," IEEE Trans. Automatic Control, vol. 61, no. 1, pp. 1-6, 2016.

[8] A. Aleksandrov and A. Zhabko, "On the asymptotic stability of solutions of nonlinear systems with delay," Siberian Mathematical Journal, vol. 53, no. 3, pp. 393-403, 2012.

[9] F. Asl and A. Ulsoy, "Analytical solution of a system of homogeneous delay differential equations via the Lambert function," in Proc. American Control Conference, (Chicago), pp. 2496-2500, 2000.

[10] V. Bokharaie, O. Mason, and M. Verwoerd, "D-stability and delayindependent stability of homogeneous cooperative systems," IEEE Trans. Automatic Control, vol. 55, no. 12, pp. 2882-2885, 2010.

[11] J. Diblik, "Asymptotic equilibrium for homogeneous delay linear differential equations with 1-perturbation term," Nonlinear Analysis, Theory, Methods \& Applicarions, vol. 30, no. 6, pp. 3927-3933, 1997.

[12] D. Efimov, A. Polyakov, E. Fridman, W. Perruquetti, and J.-P. Richard, "Comments on finite-time stability of time-delay systems," Automatica, vol. 50, no. 7, pp. 1944-1947, 2014.

[13] L. Rosier, "Homogeneous Lyapunov function for homogeneous continuous vector field," Systems\&Control Lett., vol. 19, pp. 467-473, 1992.

[14] D. Efimov and W. Perruquetti, "Oscillations conditions in homogenous systems,” in Proc. NOLCOS'10, (Bologna), pp. 1379-1384, 2010.

[15] K. Gu, K. Kharitonov, and J. Chen, Stability of Time-Delay Systems. Control Engineering, Boston: Birkhäuser, 2003.

[16] E. Fridman, Introduction to Time-Delay Systems: Analysis and Control. Basel: Birkhäuser, 2014.
[17] E. Fridman and L. Shaikhet, "Delay-induced stability of vector secondorder systems via simple Lyapunov functionals," Automatica, vol. 74, pp. 288-296, 2016.

[18] E. Fridman and L. Shaikhet, "Stabilization by using artificial delays: An LMI approach,” Automatica, vol. 81, pp. 429-437, 2017.

[19] V. Kolmanovsky and V. Nosov, Stability of functional differential equations. San Diego: CA: Academic, 1986.

[20] P. Pepe and Z.-P. Jiang, "A Lyapunov-Krasovskii methodology for ISS and iISS of time-delay systems," Systems \& Control Letters, vol. 55, no. 12, pp. 1006-1014, 2006.

[21] A. R. Teel, "Connections between Razumikhin-type theorems and the ISS nonlinear small gain theorem," IEEE Trans. Automat. Control, vol. 43, no. 7, pp. 960-964, 1998.

[22] D. Efimov and W. Perruquetti, "Homogeneity for time-delay systems," in Proc. IFAC WC 2011, (Milan), 2011. 